\title{
De la relación entre la Psicología y el trabajo a la luz de sus dimensiones coloniales
}

\author{
On the relationship between Psychology and work \\ in the light of its colonial dimensions
}

Hernán Camilo PULIDO MARTíNEZ1

\begin{abstract}
Resumen
Las herramientas propuestas en los marcos poscoloniales y decoloniales no han sido de mayor interés para los investigadores que se ocupan de la interrelación entre el conocimiento psicológico y el mundo laboral. Entonces, los estudios críticos han dejado de lado las implicaciones que tiene este conocimiento cuando "viaja" más allá de las fronteras de los países en donde se produce la Psicología. En este artículo se examinan algunas "dimensiones coloniales" que emergen de las articulaciones del "complejo psi" cuando se vincula con el mundo del trabajo en lugares en donde no se producen los objetos psicológicos, sino en donde estos son apropiados, replicados o hibridados para su uso cotidiano en los ámbitos laborales.
\end{abstract}

Palabras clave: Colonialidad; Complejo psi; Psicología crítica; Psicología de las organizaciones.

\begin{abstract}
The tools proposed in the postcolonial and de-colonial frameworks have not been of interest to researchers preoccupied with the interrelation between the psychological knowledge and the world of work. Thus, critical studies have left aside the implications that this knowledge has when it goes beyond the borders in which Psychology is produced. This article examines some "colonial dimensions" that emerged from the articulations of the "psy complex" in places that do not produce psychological objects, but which are appropriate, replicated and hybridized for being used in workplaces.
\end{abstract}

Keywords: Coloniality; Psy complex; Critical psychology; Organizational psychology.

Las herramientas propuestas en los marcos poscoloniales y/o decoloniales no han sido de mayor interés para los investigadores que se ocupan de examinar críticamente la interrelación entre el conocimiento psicológico y el mundo del trabajo. Resulta asombroso que se hayan dejado de lado estos marcos críticos cuando se hace necesario interrogar, tanto los problemas relacionados con el

\footnotetext{
1 Pontificia Universidad Javeriana, Faculta de Psicología. Edificio Manuel Briceño, Carrera 7, n 39-00, Bogotá, Colombia. E-mail: <cpulido@javeriana.edu.co>.

Apoyo: Este artículo es resultado del Proyecto ID 003917, financiado por la Vicerrectoría de Investigación de la Pontificia Universidad Javeriana, Bogotá, Colombia.
} 
viaje de la Psicología a través de los ámbitos laborales globalizados, como las contribuciones de esta disciplina a la constitución del orden internacional del trabajo y de las subjetividades que lo acompañan. La incorporación de los marcos poscoloniales y decoloniales es indispensable para dar respuestas simultaneas acerca del lugar que ocupa y de las operaciones que cumple la Psicología en los países del Atlántico Norte, en donde se producen los objetos psicológicos, como en las sociedades del Sur global, en donde estos objetos son fundamentalmente replicados, adaptados, e hibridados para su uso local (Pulido-Martínez, Garcia-Alvarez, Carvajal-Marín, \& González-Ortiz, 2013).

Posibles explicaciones de esta situación de carencia hacen referencia tanto a la manera en que se presenta la Psicología como un conocimiento científico sobre el trabajo que tendría, mediante procedimientos adaptativos, una aplicabilidad universal, como al supuesto compartido por la comunidad de psicólogos de que el conocimiento que se produce no puede tener unas dimensiones coloniales, en la medida en que sirve para el mejoramiento del trabajo y para el progreso de la sociedad (Pulido-Martínez, 2007; Staeuble, 2003, 2005). Se afirma que si la aplicación de la Psicología significa bienestar y modernización de los ámbitos en donde se labora, no habría razón para tener vinculación alguna con la colonialidad. Por el contrario, tal como se hace hasta la fecha en los países en donde no se produce Psicología, se argumentaría más bien que habría que celebrar con más ahínco la llegada de las herramientas psicológicas para intervenir y modernizar las organizaciones en donde se trabaja.

En este panorama, se propone como objetivo de este articulo presentar algunas de las dimensiones coloniales de la Psicología en su especifica relación con el mundo laboral que han sido olvidadas por la crítica. Para esto, se parte de examinar los señalamientos que en la década de los años setenta hicieron los psicólogos en América Latina acerca de las implicaciones que tiene la importación de la Psicotecnología laboral. Se consideran en seguida las posibilidades de la constitución de una Psicología autóctona del trabajo como alternativa para salir de las relaciones de colonialidad. A partir de ahí, se examinan las implicaciones que tiene el conocimiento psicológico en términos de la constitución de la subjetividad laboral subordinada $y$, finalmente, se discute la relación entre la Psicología y el trabajo en referencia a sus imbricaciones con la geopolítica del conocimiento.

\section{Un inicio para la crítica de la relación de la Psicología con el trabajo}

Cabe entonces partir de una pregunta para precisar el carácter de la relación de la Psicología con el mundo laboral. ¿Tiene el conocimiento psicológico sobre el trabajo dimensiones coloniales? La respuesta que se ha formulado por parte de algunos psicólogos ha sido afirmativa. Por ejemplo, ya en 1978 Castaño y Sánchez argumentaban que la Psicotecnología laboral responde, o mejor, se diseña para resolver los problemas específicos del trabajo de los países en donde esta se produce. Razón por la cual, cuando la Psicología viaja para su aplicación a otros contextos sociales, conlleva como consecuencia que los problemas derivados de las condiciones laborales locales se subordinen, o dejen de considerarse, en las intervenciones psicológicas. Apoyándose en las propuestas elaboradas dentro de los estudios sociales de la ciencia, Castaño y Sánchez (1978) describen una serie de consecuencias debidas al uso de instrumentos psicológicos foráneos, entre las que se cuentan el estancamiento del desarrollo de un pensamiento psicológico autóctono y la obstaculización del diseño de tecnologías adecuadas para intervenir los asuntos laborales propios. En términos generales, se podría decir que Castaño y Sánchez (1978) muestran que, a la par de la utilización de la Psicología como herramienta de intervención en ámbitos laborales localizados en el Sur, se contribuye al mantenimiento de relaciones de dependencia económica y cultural, al punto que se está cambiando la dominación militar por la subordinación tecno-científica. 
Para acudir a la solución de este tipo de impases, la situación de subordinación ha sido traducida, por algunos investigadores, al conocido debate acerca de la relevancia de las herramientas psicológicas para su uso en diferentes ámbitos culturales (Kieser \& Seidl, 2015; Long, 2013; Sher \& Long, 2012; Sloan, 1996). De manera genérica, esta discusión asume que las técnicas psicológicas necesitan de mejoras constantes a través de los procedimientos de adaptación cultural, los cuales permitirán hacer pertinente a la Psicología. Por supuesto, se considera que, por medio de las adaptaciones culturales, las dimensiones coloniales anteriormente descritas se mitigarán o desaparecerán, pues la naturaleza del conocimiento psicológico, en su calidad de saber científico, tarde o temprano eliminará los sesgos que lo atan a los centros de producción localizados en las sociedades del Atlántico Norte al incluir las debidas variaciones culturales.

Sin embargo, está bien descrito que esta forma de concebir que, con el tiempo y los debidos procedimientos adaptativos, el conocimiento psicológico y sus prácticas derivadas encajarán perfectamente en el sitio a donde llegan, abre nuevos problemas a este respecto más que solucionar las vinculaciones de la Psicología con las dimensiones coloniales (Burman, 2007). Esto ocurre en la medida en que se asume en estos procesos de adaptación que los problemas del trabajo en el norte y en el sur son cuantitativamente diferentes, pero cualitativamente iguales. Así, por ejemplo, se supone que los pequeños cambios en la forma de los instrumentos apropian las variaciones de las condiciones laborales locales. En esta forma de concebir el lugar y las operaciones de la Psicología está implícita una continuación de las categorías que dan origen a la técnica, puesto que el cambio en las categorías implicaría la pérdida de la identidad del instrumento. Esto da como resultado un efecto en el que los problemas de los lugares en donde se produce conocimiento psicológico y aquellos que lo adaptan, replican o apropian, parecerían sólo una variación en la intensidad de las categorías propuestas. Por este motivo, las diferencias culturales, económicas, sociales, históricas y, por supuesto, las diferencias que se concretan en las organizaciones particulares, desaparecen ante los resultados de la aplicación de los instrumentos psicológicos ahora adaptados. En estas condiciones está vedado, entonces, que categorías propias del mundo del trabajo en el Sur transformen o invaliden las formas de concebir el papel del conocimiento psicológico.

En la dimensión de subordinación que se produce ante la expansión de la Psicología, la producción que se lleva a cabo en el "resto del mundo" se limita, como demuestran los investigadores en los campos de la historia y de los estudios sociales de la ciencia, a hacer el aporte "exótico" al cuerpo de conocimientos existente, o a unas contribuciones que no rebasan las fronteras nacionales (Gorbach \& López Beltrán, 2008). En esta misma línea, en términos generales, y por medio de las adaptaciones culturales, se consigue un efecto insospechado: la adaptación de la Psicotecnología laboral conlleva una validación local de los marcos conceptuales, metodológicos e ideológicos presentes en las sociedades en donde se producen los objetos psicológicos. De este modo, la Psicología creada en las sociedades foráneas entra a través del interjuego con los procesos de adaptación locales, a construir y confirmar la superioridad de la ciencia importada que ahora, y a través de los procedimientos estadísticos, se torna en ciencia validada interculturalmente (Brock, 2006).

\section{La producción del conocimiento autóctono y la cuestión colonial}

Hasta aquí entonces se ha establecido de manera general que, en los países en donde se produce la Psicología, esta resulta adecuada en la medida que aporta soluciones convenientes a los problemas del trabajo. Por el contrario, en los lugares en donde la Psicología se consume como producto importado, en la medida en que subordina los problemas locales a soluciones ajenas, representa una manera en que se reproduce la colonialidad. Hay que resaltar que, aun con este lastre, está aceptado por los profesionales e investigadores que el conocimiento psicológico tiene un potencial para entender e intervenir en los problemas del trabajo. 
Así, al igual que en otras formas de ejercer la crítica, al señalar los "horrores y errores" de la Psicología en su relación con el trabajo, están abiertos los caminos para su mejoramiento (Pulido-Martínez \& Sato, 2013; Rose, 1996). Dos alternativas se han propuesto en esta situación para producir un conocimiento psicológico mejor. Por una parte, la ya mencionada serie de adaptaciones para hacer relevante a la Psicología; y, por otra, la producción de una Psicología autóctona que considere como centro los problemas locales del trabajo. Esta última opción busca optimizar la relación entre la Psicología y el trabajo a través de una "autoctonización" o "indigenización" para el Sur. Esta empresa ha llevado, por ejemplo, a las múltiples propuestas que constituyen la Psicología del trabajo de América Latina. Con esta operación de hacer pertinente este conocimiento, se han trazado soluciones a los problemas que habían dejado planteados Castaño y Sánchez en la década de los setenta (1978), y Martín-Baró en los años ochenta (1989), en referencia a la constitución de una Psicología liberadora que considerara las condiciones locales de trabajo y el papel de los menos favorecidos en el mundo laboral.

Los intentos por tener en cuenta las características del trabajo en Latinoamérica se han concentrado alrededor de incluir las condiciones objetivas de trabajo específicas de la región. De este modo, se ha dado lugar no solamente a versiones de la disciplina "autoctonizadas" o "indigenizadas", sino que, por su extensión y relevancia, han emergido verdaderas "contra-psicologías" (Parker, 2007). Es decir, han surgido perspectivas psicológicas sobre el trabajo que entran en franca lucha con el conocimiento hegemónico buscando como objetivo su reemplazo definitivo. En este horizonte se han formulado propuestas que, si bien explícitamente no se nombran como tentativas poscoloniales o decoloniales, sí se constituyen en una serie de Psicologías que tratan de entender, a partir de las condiciones propias de la organización del trabajo, lo que acontece con los seres humanos que laboran. Por lo común, la mayoría de estas perspectivas hacen apropiaciones de marcos que consideran las condiciones laborales construidas en las sociedades del Atlántico Norte, especialmente de los países de Europa, para elaborar posiciones hibridas o apropiaciones particulares. Las obras colectivas editadas por Soto Roy (2008) y Stecher (2014) que contemplan los procesos de construcción de la identidad y de la subjetividad; los estudios sobre el desgaste del trabajador llevados a cabo por Laurell (1978), así como los análisis conducidos por Coutinho, Borges, Graf, y Da Silva (2013); Bernardo (2009); Huertas Hernández (2007); Moncada Aristizabal y Burbano Valente (2005); Peralta Gómez y Bernal (2013); Pulido-Martínez y Carvajal-Marín (2005); Renteria Perez y Malvezzi (2008); Sato (2007); sobre las formas de trabajo presentes en América Latina son muestras de esta variante del conocimiento psicológico que busca superar los desaciertos para el Sur de la relación entre la disciplina psicológica y el mundo laboral.

Pero, ¿puede decirse que "realmente" se superan las dimensiones coloniales por medio de la producción de una Psicología con características más autóctonas? La respuesta puede ser positiva si se considera que las Psicologías propuestas son apropiaciones e hibridaciones que introducen las condiciones laborales como foco de análisis, las cuales, consecuentemente, persiguen proponer caminos liberadores para los trabajadores, tal como propuso la crítica de corte marxista universalista de la Psicología (Baritz, 1960; Braverman, 1974; Prilleltensky, 1994; Wexler, 1983). Sin embargo, también se podría dar una respuesta negativa en la medida en que las Psicologías "autoctonizadas" mantienen una relación de dependencia con los conceptos y prácticas propuestos en las metrópolis, en donde igualmente se producen ambos, el conocimiento psicológico crítico y el hegemónico sobre el trabajo. Las versiones "autoctonizadas", resultantes de marcos críticos, están ubicadas en los márgenes de la disciplina y responden a formas alternativas de producir el conocimiento psicológico; sin embargo, continúan manteniendo relaciones particulares de dependencia con el conocimiento, ahora crítico, que se produce en las sociedades del Atlántico Norte (Pulido-Martínez et al., 2013). En este sentido, las versiones críticas, al igual que el 
conocimiento psicológico hegemónico sobre el trabajo, suponen también un carácter universal, así como un resultado particular que ha sido llamado como la integración subordinada al conocimiento internacional (Kreimer, 2006). Por tanto, los profesionales e investigadores con tintes críticos expanden, en una relación de negativo y positivo con el conocimiento hegemónico, las formas de pensar psicológicas sobre el trabajo, contribuyendo de esta forma a la expansión de la empresa psicológica al nivel universal. De este modo, se logra dejar sin analizar minuciosamente el lugar que tiene, y las operaciones que cumple, el conocimiento psicológico y sus prácticas derivadas en las sociedades de Sur.

\section{Las dimensiones coloniales y la producción de la subjetividad}

Ahora bien, si tanto la versión hegemónica de la Psicología como las versiones críticas forman parte de la empresa psicológica universal, resultan, entonces, de muy diversas maneras complementarias a la hora de expandir las formas de pensar psicológicas. Es precisamente esta complementariedad de las formas que asume la relación de la Psicología con el trabajo uno de los aspectos que le imprime su plasticidad para adaptarse a los cambios en la organización del trabajo y producir al sujeto trabajador que se demande (Pulido-Martínez, 2015). Como sugieren una pléyade de investigadores, entre los que se cuenta Hollway (1991); Rose (1999); Townley (1994); y Walkerdine (2005) contemporáneamente, las maneras de concebir e intervenir al trabajo y a los trabajadores pasan por algún tipo de Psicología. Entonces, si se tiene en cuenta que la Psicología construye al sujeto cuando habla de este, no habría que preguntarse si la producción de la subjetividad entra dentro de las dimensiones coloniales de la Psicología, sino más bien hasta qué punto esas dimensiones son constitutivas de la relación de la Psicología con el trabajo.

Se puede señalar, siguiendo las pistas propuestas en el trabajo de Hollway (1998), que la producción de subjetividad mediante la aplicación de los discursos y prácticas psicológicas ocurre independientemente de los tipos de Psicologías que se pongan en circulación, de lo pertinentes que ellas resulten y de la variedad de instrumentos que se utilicen. El resultado es un efecto de subjetividad consistente en que la práctica psicológica, en el caso que fuere, contribuye a la construcción del sujeto psicológico moderno que la producción y reproducción de la sociedad liberal capitalista requiere.

No se trata, entonces, de establecer los casos afortunados en donde se hizo la aplicación de la Psicología que dieron como resultado el alcance de los objetivos para los que fue propuesta. Es en cada aplicación de la Psicología que se produce y reproduce al sujeto psicológico indispensable para que el capitalismo y la sociedad liberal se perpetúen. Las incoherencias que frecuentemente se señalan en las actividades de los departamentos de recursos humanos, la "tropicalización" de las estrategias psicológicas, la trivialización del trabajo y la cualificación constante del trabajador en términos psicológicos, adecuados a las transformaciones del mundo laboral, son todas contribuciones del "complejo psi" a la constitución del sujeto psicológico contemporáneo (Ingleby, 1985; Pulido-Martínez \& Carvajal-Marín, 2016; Rose, 1985; Walkedine, 2005).

Algunos estudios que se han hecho acerca de las articulaciones amplias del "complejo psi" muestran, como había anotado Rose (1996), las maneras de producción de subjetividad laboral en el interjuego de las Psicologías. Así, contemporáneamente, las condiciones asociadas al empleo se han vuelto problemáticas ante el avance de las prescripciones neoliberales que proponen la eliminación de las certidumbres sociales que cobijaban a los trabajadores. Por medio del examen de la articulación entre las formas psicológicas de concebir al trabajador, su salud, el riesgo en el trabajo y al trabajo como riesgo, Pulido-Martínez y Carvajal-Marín (2016) describieron cómo en la actualidad el empleo y las condiciones que lo acompañan se convierten en factores patologizantes, mientras que las condiciones flexibles de trabajo y los riesgos a los que se compele al trabajador para 
que los asuma se tornan en factores promotores de salud. El ensamblaje que se efectúa se lleva a cabo entre las Psicologías de corte humanista y positivista con aquellas de matices constructivistas que, como ha sido señalado en la literatura, responden a las formas fordistas y posfordistas de organización del trabajo (Brinkmann, 2008). Entre las consecuencias que vienen de la mano con estas formas en las que se ensambla la relación de la Psicología con el trabajo se cualifica y homogeniza al trabajador alrededor del mundo y se facilita, de esta forma, el ejercicio de gobierno de la fuerza laboral a los niveles internacionales.

Se debe anotar aquí que desde que la Psicología estableció su relación con el mundo del trabajo, tomó implícitamente, como centro de su reflexión e intervención, a la figura del trabajador libre que emergió dentro de la economía política. A lo largo de la historia, la Psicología ha calificado esta figura, inicialmente, como un trabajador libre y sentimental (Hollway, 1991); y en tiempos de flexibilización y precarización como los que corren, ha vuelto a cualificar a este trabajador libre no solamente en los términos humanistas, sino ahora también lo concibe como aquel trabajador "corcho", "portafolio" o "disponible" que las formas de organizar el trabajo que requiere la geocultura liberal (Sisto, 2009).

La producción de la subjetividad del trabajador en referencia a las dimensiones coloniales por supuesto no se detiene aquí. También está involucrada la producción del "otro" de este trabajador ideal que, en términos psicológicos, se construyó en las sociedades del Atlántico norte. En esta línea, mediante el uso de la Psicología, y en una relación de cara-contracara, emergen diferentes versiones del sujeto que trabaja, las cuales conectan los procesos locales del trabajo con las prescripciones globales que se formulan para su regulación. En términos de ilustrar esta manera de operar de la Psicología, es posible mencionar cómo los trabajadores que no están vinculados con las organizaciones que responden a la organización del trabajo que impera, se les ubica fácilmente en la posición de aquellos que tienen que cambiar, pues retrógrados, subdesarrollados, atrasados, tradicionales, negados al cambio o carentes de criterio para aceptar la modernización. En ultimas, se les constituye como sujetos que deben ser intervenidos en términos psicológicos para mejorar su bienestar y restablecer su humanidad.

A este respecto, de cómo con base en la Psicología se producen efectos de subjetividad entre diferentes regiones del planeta, se ha examinado cómo han sido prescritas las formas de ser y de comportarse de los trabajadores alrededor del mundo (Vargas Monroy \& Pujal I Llombart, 2013). En esta vía, Pulido-Martínez (2007) mostró que, mientras que en los Estados Unidos, durante los años del desarrollismo, los psicólogos crearon en la jerga psicológica al ciudadano trabajador, en América Latina los psicólogos construyeron como contracara, en los términos psicológicos opuestos, a la figura de los pobres de la región. A partir de aquí se formularon una serie de acciones a nivel internacional que tenían como objeto la transformación de la "mente" de los habitantes del Sur, pues se consideró que la falta de desarrollo radicaba de manera fundamental en las características psicológicas que les eran atribuidas a los habitantes pobres de las sociedades del Sur. Sobra anotar que, apoyadas en este tipo de construcciones duales de la subjetividad, las entidades regulatorias internacionales vehiculizan políticas, imponen planes y se ejercen acciones que afectan a poblaciones enteras de trabajadores alrededor del mundo, a las cuales pocos se oponen pues están construidas en el lenguaje de la ciencia psicológica universal.

\section{Geopolítica y Psicología en el trabajo}

Muchos de los aspectos considerados hasta aquí muestran cómo la Psicología guarda una serie de relaciones entre los lugares de producción y aquellos sitios a los cuales llega para ser utilizada. Este viaje de la Psicología no es una cuestión puramente de trasplante útil, sino que van allí involucrados una serie de aspectos que puede decirse responden a una geopolítica del conocimiento 
psicológico (Molinari, 2004). Por geopolítica del conocimiento psicológico se entiende, por una parte, que los objetos de esta disciplina tienen un lugar geográfico de nacimiento; y, por otra, que el conocimiento psicológico que se produce en esos sitios, al viajar, se halla inmerso en relaciones desiguales de poder en donde quienes lo generan se encuentran en condiciones de ventaja frente a aquellos que lo usan. En este sentido, los apartados de este texto pueden verse como aspectos de una lectura de las manifestaciones de la geopolítica de la Psicología que presenta relaciones con la colonialidad.

Por supuesto, a este respecto vale anotar que la geopolítica que se establece alrededor de la relación entre la Psicología y el trabajo no es nueva. En la medida en que los psicólogos, como otros científicos sociales, consideraron desde comienzos del siglo XX que la sociedad liberal capitalista era el culmen del desarrollo humano digno de extenderse por doquier, y que la Psicología era básica para la perpetuación de su compañero, el capitalismo, (Viteles, 1932), la empresa psicológica - en relación con el trabajo -, no se impregnó de un carácter liberador, más bien se convirtió en un proyecto civilizatorio y por ende colonizador (Pulido-Martínez, 2011). Solamente a manera de ejemplo y con base en la Psicología se sentaron los cimientos para poder prescribirle al planeta cómo se debía gobernar el mundo del trabajo en términos de "la mejor manera" para administrarlo. Esta "mejor manera", generalmente, estaba representada por el conocimiento procesado en las facultades de Administración y Psicología de las universidades en los Estados Unidos. Obviamente, no es esta una imposición con el uso directo de la fuerza; es más bien, como señala Van Strien (1997) una sumisión intelectual voluntaria que recorre el mundo. Esta sumisión puede ser vista como el resultado de la mezcla entre la seducción inevitable de lo que se presenta como la forma más adecuada y moderna de administrar, en conjunto con el olvido de las condiciones objetivas en las que se trabaja y el éxito en la producción de la subjetividad del trabajador. Es quizá esta combinación lo que lleva a pedir más y más Psicología en los ámbitos laborales y a evitar que otras formas de pensamiento sobre el trabajo entren en competencia.

Contemporáneamente, la geopolítica en términos de producción y distribución de la Psicología tiene particularidades específicas. De acuerdo con Moghaddam (1987), se han construido tres mundos de la Psicología: el primer mundo está constituido por la producción que se realiza en los Estados Unidos, la cual se ha posicionado como el conocimiento psicológico hegemónico; al segundo mundo pertenecen los países europeos como el Reino Unido, Alemania y Francia, quienes entran en franca competencia con la producción norteamericana. Y por último se encuentran los países que son habidos consumidores de Psicología que componen el resto del mundo. Así se constituyen y manifiestan una serie de diferencias en la producción y el consumo de la Psicología que, según Moghaddam y Lee (2006), responden más al poderío militar y a la posición política de los Estados Unidos que al virtuosismo del conocimiento psicológico.

Puede decirse que geopolítica y colonialidad de la Psicología son dos caras de una misma moneda, que está a la base de determinadas formas de ser y estar en el trabajo al nivel mundial. La salida de la condición en la cual regiones enteras del planeta están, como señala Ibarra Colado (2006), mirándose a sí mismas en el espejo del colonizador, está a la orden del día. Una certeza ha sido prescrita, la solución a las dimensiones coloniales del conocimiento psicológico difícilmente provendrá de las "ciencias psi", puesto que ellas mismas están involucradas en la producción del colonialismo (Penson, 2014).

\section{Conclusión}

Después de constatar que las relaciones de colonialidad están presentes en el vínculo entre la Psicología y el trabajo, no se puede seguir pensando de la misma manera. Hay que cuestionar el conocimiento psicológico sobre el trabajo, más que tratar de mejorarlo. Quizá este examen deba llevarse a cabo en términos de una reflexión histórica que dé cuenta de cómo se ha llevado a cabo un proceso 
de psicologización tan intensa, como aquel que está ocurriendo en los países de América Latina. No hay que olvidar que, en estas regiones subordinadas, es en donde la disciplina crece con mayor velocidad y donde todos los días aumenta el número de psicólogos que también va a actuar en el mundo del trabajo.

Hay que mencionar que una, se podría llamar actitud, ha caracterizado a la disciplina psicológica y a los psicólogos mismos en relación con el mundo laboral. Esta actitud puede ser descrita como una "correcta ignorancia", la cual hace referencia al puesto que tienen las múltiples críticas que se formulan en contra del pensar y actuar psicológico en el trabajo (Pulido-Martínez \& Sato, 2013). La "correcta ignorancia" que se incrementa a medida que se psicologizan los ámbitos laborales produce que las criticas tengan un "no lugar" dentro de la relación que la Psicología ha establecido con el trabajo. Contraria a otras ramas de la Psicología, como señala Teo (2005), que avanzan a través de las críticas, en la relación conocimiento psicológico-mundo del trabajo esto parece no ocurrir. Las críticas se mantienen marginales, se les hace poco caso y no propician nuevos desenvolvimientos; por el contrario, se produce más de lo mismo sin mayores miramientos. En otras palabras, en la relación que la Psicología ha establecido con el trabajo se presenta poca reflexión acerca del sentido del actuar psicológico más allá del sesgo pro-gerencia. La pregunta técnica sobre cómo aumentar la productividad impera, y la trivialización del trabajo en términos psicológicos aumenta constantemente. Por supuesto, no es por la coherencia o pertinencia de las críticas que esto ocurre. La negación o el relegamiento al que se ven sometidas las críticas formuladas se lleva a cabo por parte de los profesionales e investigadores, así como también por las facultades de Psicología.

Ya hace muchas décadas, Canguilheim (2011) había señalado que la pregunta ¿qué es la Psicología? dejaba en una situación muy incómoda a la disciplina. La pregunta contemporánea ¿qué hace la Psicología? en los ámbitos institucionales y en el trabajo en particular, quizá la deja en una peor "correcta ignorancia" que impera, pues no resulta pertinente, no al menos, para aquellos lugares en donde este conocimiento llega como producto importado.

\section{Referencias}

Baritz, L. (1960). The servants of power. A history of the use of social science in. Middletown: Wesleyan University Press.

Bernardo, M. H. (2009). Trabalho duro: discurso flexível. São Paulo: Expressão Popular.

Braverman, H. (1974). Labor and monopoly capital. The degradation of work in the twentieth century. New York: Monthly Review Press.

Brinkmann, S. (2008). Changing psychologies in the transition from industrial society to consumer society. History of the Human Science, 21(2), 85-119. http:// dx.doi.org/10.1177/0952695108091412

Brock, A. (2006). Internationalizing the history of psychology. New York: New York University Press.

Burman, E. (2007). Between orientalism and organization. Cross cultral lesson from Japan for a critical history of psychology. History of Psychology, 10(2), 179-198. http://dx.doi.org/10.1037/1093-4510.10.2.179

Canguilhem, G. (2011). Estudios de historia y de filosofía de las ciencias. Buenos Aires: Amorrortu.

Castaño, D., \& Sánchez, G. (1978). Problemas de la importacion psicologica tecnológica psicolaboral en los paises en desarrollo. Revista Latinonamericana de Psicología, 10(1), 71-82.

Coutinho, M. C., Borges, R. C., Graf, L. P., \& Da Silva, A. S. (2013). "Todo dia en uma casa diferente": Entre trajetórias, sentidos e o cotidiano laboral de diaristas. Universitas Psychologica, 12(4), 1125-1138. http://dx. doi.org/10.11144/Javeriana.UPSY12-4.tdcd

Gorbach, F., \& López Beltrán, C. (2008). Saberes locales. Ensayos sobre historia de la ciencia en America Latina. Michoacan: El Colegio de Michoacan.

Hollway, W. (1991). Work psychology and organizational behaviour. London: Routledge.

Hollway, W. (1998). Fitting work: Psychological assessment in organizations. In J. Henriques, W. Hollway, C. Urwin, C. Venn, \& V. Walkerdine. Changing the subject. Psychology, social regulation and subjectivity (pp.24-57). London: Routledge.

Huertas Hernández, O. L. (2007). La narrativa como posiblidad de comprensión de las organizaciones productivas rurales. Universitas Psychologica, 6(1), 163-172.

Ibarra Colado, E. (2006). Organization studies and epistemic coloniality in Latin America: Thinking 
otherness from the margins. Organization, 13(4), 463-488. http://dx.doi.org/10.1177/1350508406065 851

Ingleby, D. (1985). Professionals as socializers: The 'psy complex'. Research in Law, Deviance and Social Control, 7, 79-109.

Kieser, A. N., \& Seidl, D. (2015). The practical relevance of management research: Turning the debate on relevance into a rigorous scientific research program. Academy of Management Annals, 9(1), 143-233. http://dx.doi.org/10.1080/19416520.2015.1011853

Kreimer, P. (2006). ¿Dependientes o integrados? La ciencia Latinoamericana y la nueva división internacional del trabajo. Nomadas, 24, 194-212.

Laurell, A. C. (1978). Proceso de trabajo y salud. Cuadernos Políticos, 17, 59-79. Acceso el Noviembre 4, 2011, de http://www.cuadernospoliticos.unam.mx/ cuadernos/contenido/CP.17/17.7.AsaCristina.pdf

Long, W. (2013). Rethinking relevance. South African psychology in context. History of Psychology, 16(1), 19-35. http://dx.doi.org/10.1037/a0029675

Martín-Baró, I. (1989). Psicología política del trabajo en América Latina. Revista de Psicología de El Salvador, $8(31), 2-25$.

Moghaddam, F. (1987). Psychology in the three worlds: As reflected by the crisis in social psychology and the move toward indigenous psychology. American Psychologist, 42(10), 912-920.

Moghaddam, F. M., \& Lee, N. (2006). Double reification: The processes of universalizing psychology in the three worlds. In A. Brock. Internationalizing the history of psychology (pp.163-182). New York: New York University Press.

Molinari, J. M. (2004). Hacia una nueva geopolítica de la psicología: Prospectiva, conceptos y tendencias. Acta Psiquiátrica y Psicológica de América Latina, 50(1), 25-33.

Moncada Aristizabal, C. A., \& Burbano Valente, J. (2005). Modelo de estructura empresarial para el transporte público colectivo. Universitas Psychologica, 4(3), 325-337.

Parker, I. (2007). Revolution in psychology. Alienation to emancipation. London: Pluto Press.

Penson, W. J. (2014). "Psy-science and the colonial relationship in the mental health field". Mental Health Review Journal, 19(3), 176-184. http://dx.doi.org/10. 1108/MHRJ-03-2014-0006

Peralta Gómez, M. C., \& Bernal, C. A. (2013). “No quiero que usted sea asi". Macro y micro discursos que posicionan a los sujetos laborales que trabajan en las calles de Bogotá. Universitas Psychologica, 12(4), 1139-1152. http://dx.doi.org/10.11144/Javeriana.UP SY12-4.nqqu

Prilleltensky, I. (1994). The morals and politics of psychology. New York: State Universty of New York Press.
Pulido-Martínez, H. C. (2007). Produciendo trabajadores modernos: Conocimiento psicológico y el mundo del trabajo en el Sur. Universitas Psychologica, 6(1), 27-37.

Pulido-Martínez, H. C. (2011). Psicología y trabajo: Una relación bajo examen. En B. P. Ballesteros de Valderrama \& H. Escobar Melo. Psicología y políticas públicas: Aportes desde la academia (pp.123-144). Bogota: Editorial Pontificia Universidad Javeriana.

Pulido-Martínez, H. C. (2015). Del empleo al post-empleo: O de la plasticidad de la psicología en la produccion de la subjetividad laboral. Revista Psicologia: Organizações e Trabalho, 15(3), 322-331. http://dx.doi.org/ 10.17652/rpot/2015.3.508

Pulido-Martínez, H. C., \& Carvajal-Marín, L. M. (2005). De la tradición a la racionalización. Repertorios culturales que circulan en una organización alimentadora del sistema de transporte masivo de pasajeros en Bogotá. Universitas Psychologica, 4(3), 349-357.

Pulido-Martínez, H. C., \& Carvajal-Marín, L. M. (2016). Patologizacion y despatologizacion de la ficcion del "trabajador libre" en terminos del riesgo psico-laboral. En A. A. Leal Ferreira, A. Molas, \& J. Carrasco. Psicologia, tecnologia e sociedade: Controvérsias metodológicas e conceituais para uma análise das práticas de subjetivação (pp.239-258). Rio de Janeiro: Nau.

Pulido-Martínez, H. C., \& Sato, L. (2013). ...Y entonces ¿esto de la critica que es? De las relaciones entre la psicologia y el mundo del trabajo. Universitas Psychologica, 12(4), 1355-1368. http://dx.doi.org/ 10.11144/Javeriana.UPSY12-4.rpmt

Pulido-Martínez, H. C., Garcia-Alvarez, C. M., CarvajalMarín, L. M., \& González-Ortiz, I. D. (2013). Entre bendiciones y perjuicios. Mapeando el lugar y las operaciones de la psicología en relacion el trabajo en el sector de la salud. En O. Restrepo. Ensamblando estados. Proyecto ensamblado en Colombia (pp.355-370). Bogota: Panamericana Formas e Impresos.

Renteria Perez, E., \& Malvezzi, S. (2008). Empleabilidad, cambios y exigencias psicosociales en el trabajo. Universitas Psychologica, 7(2), 319-334.

Rose, N. (1985). The psychological complex. Psychology, politics and society in England, 1869-1939. London: Routledge.

Rose, N. (1996). Inventing ourselves: Psychology, power and regulation. London: Sage.

Rose, N. (1999). Governing the soul. The shaping of the private self. London: Free Association Books.

Sato, L. (2007). Procesos cotidianos de organização do trabalho na feira livre. Psicologia \& Sociedade, 19(1), 95-102. http://dx.doi.org/10.1590/S0102-71822007 000400013 
Sher, D., \& Long, W. (2012). Historicising the relevance debate: South African and American psychology in context. South African Journal of Psychology, 42(4), 564-575. http://dx.doi.org/10.1177/0081246312042 00410

Sisto, V. (2009). Cambios en el trabajo, identidad e inclusion social en Chile: Desafios para la investigación. Universum, 24(2), 192-216. http://dx.doi.org/10.4067/ S0718-23762009000200011

Sloan, T. S. (1996). Psychological research methods in developing countries. In S. C. Carr \& J. F. Schumaker. Psychology and the developing world (pp.38-45). Westport: Praeger.

Soto Roy, A. (2008). Flexibilidad y subjetividades. Hacia una comprensión psicosocial del empleo contemporáneo. Santiago de Chile: LOM.

Staeuble, I. (2003). De-centring Western perspectives: Psychology and the disciplinary order in the firstand third world. In A. C. Brock, J. Low, \& W. Van Hoorn (Eds.), Rediscorvering the history of psychology: Essays inspired by the work of Kurt Dazinger (pp.183-206). New York: Kluwer Academic.

Staeuble, I. (2005). The international expansion of psychology: Cultural imperialism or chances for alternative cultures of knowledge. In A. Gulerce, A. Hofmesiter, I. Staeuble, G. Saunders, \& J. D. Kay. Contemporary theorizing in psychology: Global perspectives (pp.88-96). Concord: Captus University.

Stecher, A. (2014). El campo de investigación sobre transformaciones del trabajo, identidades y subjetividad en la modernidad contemporanea.
Apuntes desde Chile y America Latina. En A. Stecher \& L. Godoy. Transformaciones del trabajo, subjetividad e identidades: Lecturas psicosociales desde Chile y America Latina (pp.19-76). Santiago de Chile: Ril Editores.

Teo, T. (2005). The critique of psychology: From Kant to postcolonial theory. Toronto: Springer.

Townley, B. (1994). Refraiming human resources management: Power, ethics and the subject at work. London: Sage.

Van Strien, P. J. (1997). The American "colonization" of Northwest European social psychology after World War II. Journal of the History of the Behavioral Sciences, 33(4), 349-363. http://dx.doi.org/10.1002/ (SICI)1520-6696(199723)33:4<349::AID-JHBS1> 3.0.CO:2-K

Vargas Monroy, L., \& Pujal I Llombart, M. (2013). Gubernamentalidad, dispositivos de género, raza y trabajo: La conducción de la conducta de las mujeres trabajadoras. Universitas Psychologica, 12(4), 1225-1267. http://dx.doi.org/10.11144/Javeriana.UPSY12-4.gdgt

Viteles, M. S. (1932). Motivation and morale in industry. London: Staples Press Limited.

Walkerdine, V. (2005). Freedom, psychology and the neoliberal subject. Soundings, 25, 7-61.

Wexler, W. (1983). Critical social psychology. London: Routledge.

Recibido: Mayo 16, 2016

Versión final: Julio 7, 2016

Aprobado: Agosto 15, 2016 